\title{
Cocoa procyanidins modulate transcriptional pathways linked to inflammation and metabolism in human dendritic cells
}

Article

Accepted Version

Midttun, H. L. E., Ramsay, A., Mueller-Harvey, I. and Williams, A. R. (2018) Cocoa procyanidins modulate transcriptional pathways linked to inflammation and metabolism in human dendritic cells. Food \& Function, 5. pp. 2883-2890. ISSN 2042650X doi: https://doi.org/10.1039/C8FO00387D Available at https://centaur.reading.ac.uk/76829/

It is advisable to refer to the publisher's version if you intend to cite from the work. See Guidance on citing.

To link to this article DOI: http://dx.doi.org/10.1039/C8FO00387D

Publisher: Royal Society of Chemistry

All outputs in CentAUR are protected by Intellectual Property Rights law, including copyright law. Copyright and IPR is retained by the creators or other copyright holders. Terms and conditions for use of this material are defined in the End User Agreement. 


\section{CentAUR}

Central Archive at the University of Reading

Reading's research outputs online 


\title{
Cocoa procyanidins modulate transcriptional pathways linked to inflammation and metabolism in human dendritic cells
}

\author{
Helene L.E. Midttun ${ }^{1}$, Aina Ramsay ${ }^{2}$, Irene Mueller-Harvey², Andrew R. Williams ${ }^{1 *}$ \\ ${ }^{1}$ Department of Veterinary and Animal Sciences, University of Copenhagen, Frederiksberg, Denmark \\ ${ }^{2}$ Chemistry and Biochemistry Laboratory, School of Agriculture, Policy and Development, University of Reading, Reading, \\ United Kingdom \\ *arw@sund.ku.dk
}

\begin{abstract}
Foods rich in polyphenols such as procyanidins (PC) have been proposed to have anti-inflammatory properties, and we have previously reported inhibition of lipopolysaccharide (LPS)-induced inflammatory cytokine secretion in human dendritic cells (DCs) by PC derived from cocoa. To explore the mechanistic basis of this inhibition, here we conducted transcriptomic analysis on DCs cultured with either LPS or LPS combined with oligomeric cocoa PC. Procyanidins suppressed a number of genes encoding cytokines and chemokines such as CXCL1, but also genes involved in the cGMP pathway such as GUCY1A3 (encoding guanylate cyclase soluble subunit alpha-3). Upregulated genes were involved in diverse metabolic pathways, but notably two of the four most upregulated genes ( $N M B$, encoding neuraminidase $B$ and $A D C Y 3$, encoding adenyl cyclase type 3) were involved in the cAMP signalling pathway. Gene-set enrichment analysis demonstrated that upregulated gene pathways were primarily involved in nutrient transport, carbohydrate metabolism and lysosome function, whereas down-regulated gene pathways involved cell cycle, signal transduction and gene transcription, as well as immune function. qPCR analysis verified differential expression of GUCY1A3, $A D C Y 3, N M B$ as well as a number of other genes, and marked suppression of LPS-induced CXCL1 and IL-23 protein secretion was also observed. Thus, our results confirm a marked anti-inflammatory effect of PC in human DCs, which may be related mechanistically to secondmessenger function and metabolic activity. Our results provide a foundation to further investigate metabolic pathways altered by PC during intestinal inflammation, and further encourage investigation of the health-promoting potential of PC-rich functional foods.
\end{abstract}




\section{Introduction}

Procyanidins (PC) are a class of bioactive plant polyphenols commonly found in dietary sources such as cocoa, grapes, nuts and berries. Procyanidins and other related polyphenols have been the object of much investigation due to their putative health-promoting properties, such as improving cardiovascular health, reducing oxidative stress, and regulating gastrointestinal inflammation and metabolic function. ${ }^{1-4}$ The mechanisms whereby PC may exert bioactivity have not been fully elucidated, but in in vitro studies isolated PC molecules have been shown to have strong antioxidant activity and reduce inflammatory responses in epithelial cells and macrophages by inhibiting toll-like receptor (TLR) induced pathways, including reduction of mitogen-activated protein kinase activity and NF-KB translocation, resulting in reduced production of inflammatory cytokines such as IL-1 $\beta .{ }^{5-10}$

The molecular structure of PC can vary widely depending on the degree of polymerization (DP) of the constituent catechin and epicatechin flavan-3-ol units (Figure 1), which may give rise to molecules ranging from dimers to polymers with a DP $>20 .{ }^{11}$ Anti-inflammatory activity has been shown to be associated with oligomers and polymers, with fractions containing these higher-molecular weight compounds efficiently inhibiting inflammatory responses in macrophages and Caco-2 cells, where monomers and lower-weight compounds had no effect. ${ }^{5,6}$ As the absorption of PC with a DP >2 in the small intestine appears to be minimal ${ }^{12}$, the modulatory effects of dietary PC likely arise through direct interactions with innate gut immune cells residing in the mucosa, although phenolic acids that are derived from the microbial breakdown of PC in the colon and subsequently absorbed may also have systemic immuno-modulatory activity. ${ }^{13,14}$ Dendritic cells (DCs) are one of the key innate immune cell populations residing at mucosal surfaces as they have the ability to sample dietary or microbial compounds either directly from the intestinal lumen, or after translocation of antigens by M-cells to the lamina propria. ${ }^{15-17}$ Subsequently, they initiate immune and inflammatory responses by secreting cytokines that induce proliferation and modulate activity of T-cells. ${ }^{18}$ Thus, modulatory effects of PC on DC function may be important in their putative anti-inflammatory activity.

We recently reported that the activity of human DCs was markedly altered after exposure to purified cocoa PC fractions, with a dramatic reduction in inflammatory cytokine production induced by the TLR4 agonist lipopolysaccharide (LPS). Consistent with previous studies, this was dependent on DP with the activity residing in the fraction containing PC with DP $>4 .{ }^{19}$ Here, we conducted transcriptomic analysis of LPS-primed human DCs cultured with or without this bioactive cocoa PC fraction, in order to gain an insight into the mechanisms underlying the modulatory activity. 


\section{Methods}

\section{Procyanidins}

Cocoa PC were purified as described previously. ${ }^{20,21}$ Briefly, cocoa beans were ground, defatted with hexane, and freeze-dried. The resulting powder was extracted with acetone/water (70:30), freeze-dried again, dissolved in water and then fractionated over a Sephadex LH-20 column. Fraction 2 was eluted with acetone/water (80:20), as shown in Supplementary File 1. HPLC-MS was performed using an HPLC Agilent 1100 series system consisting of a G1379A degasser, G1312A binary pump, a G1313A ALS autoinjector, a G1314A VWD UV detector and a G1316A column oven and API-ES instrument (Hewlett Packard 1100 MSD Series, Agilent Technologies, Waldbronn, Germany) using an ACE C18 column (3 mm; 25064.6 mm; Hichrom Ltd; Theale; UK) fitted with ACE guard column at room temperature. The gradient program employed was 1\% acetic acid in MilliQ water (solvent A) and HPLC-grade acetonitrile (solvent B) as follows: 0-35 min, 36\% B; 35-40 min, 36-50\% B; 40-45 min, 50-100\% B, which was followed by 45- 55 min, 100-0\% B; 55-60 min, $0 \% \mathrm{~B}$. The mean DP of PC in this fraction was $5.6 \pm 0.1$ (mean \pm SEM) , and the purity was $76 \%$, as deduced by thiolysis and HPLC-MS analysis. ${ }^{20}$

\section{Culture and stimulation of DCs}

Buffy coats (leukocyte-containing fraction of blood samples) were obtained from anonymous donors at Rigshospitalet, Copenhagen, Denmark, following written informed consent. PBMCs were isolated using Histopaque 1.077 (Sigma-Aldrich), and monocytes were then purified using anti-CD14 microbeads and magnetic separation (MACS, Miltenyi Biotech). Monocytes were seeded in RPMI 1640 media containing 10\% foetal calf serum, $100 \mathrm{U} / \mathrm{mL}$ penicillin, $100 \mu \mathrm{g} / \mathrm{mL}$ streptomycin, and $12.5 \mathrm{ng} / \mathrm{mL}$ each of recombinant human GM-CSF and IL-4 ( $R$ and D systems, UK). After four days differentiation, maturation was induced with 10 $\mathrm{ng} / \mathrm{mL}$ LPS (Sigma-Aldrich). Cocoa PC (10 $\mathrm{\mu g} / \mathrm{mL}, \mathrm{w} / \mathrm{v})$, or PBS as a control, were added 15 minutes prior to the addition of LPS. The concentration of PC was chosen as it resulted in no toxicity to the DCs, and was estimated to represent physiological concentrations of PC that would be found in the human intestinal lumen following normal intake of PC-rich foods. ${ }^{19,22,23}$. Cells were harvested after 6 hours for microarray and qPCR analysis, and medium was harvested after 24 hours for ELISA. Experiments were performed a minimum of three times, each time using cells from a different blood donor.

\section{Microarray and gene pathway analysis}

RNA was extracted using RNAeasy minikits (Qiagen), and quality was assessed using the Experion platform (Biorad). Labelling, hybridization and scanning was carried out using the Illumina HT-12 v4.0 platform (AROS 
Biotech A/S, Denmark). Data were quantile normalized and log2 transformed prior to statistical analysis using statistical analysis for microarrays. ${ }^{24}$ For the statistical analysis, only genes significantly expressed in at least one of the treatment groups were included, and predicted genes and genes with no gene symbol were removed. Gene set enrichment analysis (GSEA) was used for pathway analysis (Broad Institute, http://software.broadinstitute.org) using canonical pathways v5.1. Manual inspection of the KEGG, REACTOME and PID databases was carried out at www.genome.jp/kegg, htpps://reactome.org, and www.ndexbio.org, respectively. Microarray data is available at GEO under accession number GSE102895.

$q P C R$

RNA was extracted as above, and cDNA synthesis performed using qScript SuperMix (Quanta Biosciences). Primer sequences are listed in Table 1. qPCR was performed using 2X PerfeCTa SYBR Green fastmix (Quanta Biosciences). The following qPCR programme was used: an initial denaturation step at $95^{\circ} \mathrm{C}$ for 2 minutes followed by 40 cycles of 15 seconds at $95^{\circ} \mathrm{C}$ and 20 seconds at $60^{\circ} \mathrm{C}$. RPLPO was used as a reference gene for normalization. Expression was calculated as copy number based on values relative to the quantity of the reference gene.

ELISA

IL-23 concentrations were determined using commercial paired antibodies (Thermo Fisher) and CXCL1 concentrations were determined using a commercial ELISA kit (Quantakine, R and D systems) according to the manufacturer's instructions.

\section{Statistical Analysis}

Where indicated, ANOVA followed by Dunnett's multiple comparison test or two-tailed t-tests were carried out using Graphpad prism v7.0 (Graphpad Software, San Diego, CA). Significance was taken at $P<0.05$. 


\section{Results and Discussion}

\section{Transcriptomic analysis of DCs exposed to procyanidins}

We have previously shown that cocoa PC suppress the LPS-induced secretion of IL-6 and IL-12p70, whilst enhancing secretion of the regulatory cytokine IL-10. ${ }^{19}$ To explore in greater depth the intracellular pathways modulated by PC, microarray analysis was performed on DCs incubated in LPS for 6 hours with or without PC. Procyanidins significantly modulated the expression of 150 genes (121 upregluated and 36 downregulated; Supplementary File 2). The top up- and down-regulated genes are shown in Figure 2. Downregulated genes included the inflammatory chemokines CCL1, CXCL1 and CCL19, as well as the adrenergic receptor $A D R A 1 B$, the expression of which we have also shown to be suppressed in DCs by antiinflammatory products from the helminth parasite Ascaris suum. ${ }^{25}$ Also suppressed was GUCY1A3, which encodes the enzyme guanylate cyclase soluble subunit alpha-3, involved in catalysing production of cyclic guanosine monophosphate (cGMP). The most upregulated gene was SPINK1, encoding a serine protease inhibitor involved in trypsin inhibition. ${ }^{26}$ Also strongly upregluated were $N M B$ and $A D C Y 3$, encoding neuromedin B and adenylyl cyclase type 3, two proteins involved in the production and function of the second-messenger cyclic adenosine monophosphate (CAMP). The upregulation of both $N M B$ and $A D C Y 3$ suggests an increase in CAMP formation, which would be consistent with reports that cAMP suppresses inflammatory function in human DCs. ${ }^{27}$ In contrast, activation of the cGMP/cGMP kinase pathway leads to pro-inflammatory and migratory activity in DCs. ${ }^{28}$ Thus, our results suggest that modulation of intracellular second-messenger activity may contribute to the anti-inflammatory properties of PC. Most of the other upregulated genes (e.g. SLC39A11 and MMP10) were also involved in biochemical processes such as zinc homeostasis and extracellular matrix remodelling ${ }^{29,30}$, suggesting a profound effect of PC on cellular metabolism of DCs.

\section{qPCR and ELISA validation}

To confirm the results of the microarray, we conducted qPCR on selected genes indicated in Figure 2, as well as PTGS2 (encoding cyclooxygenase 2). qPCR confirmed that GUCY1A3, CXCL1 and PTGS2 were all significantly down-regulated, whilst $A D C Y 3$, SPINK1 and NMB were all up-regulated (Figure $3 \mathrm{~A}$ ). We further confirmed the suppression of CXCL1 and IL-23 by measuring secreted protein levels by ELISA, and confirmed significant suppression of both (Figure 3B).

\section{Gene pathway analysis}


To investigate gene pathways modulated by PC, gene ontogeny and pathway analysis was carried out. A number of pathways were modulated by PC treatment (Supplementary File 3 ). The top ten up-regulated gene pathways identified by GSEA are shown in Figure 4A. Significantly regulated pathways (nominal $P$ value $<0.01$, Q-value $<0.25$ ) were used for interrogation of the KEGG and REACTOME databases, which indicated that they were primarily involved in small molecule transport and carbohydrate metabolism (Figure 4B). Gene pathways connected to lysosomal metabolic function were particularly enhanced, which may be consistent with our previous data that proanthocyanidins appear to be trafficked to the lysosome after endocytosis by DCs. ${ }^{19}$ Inspection of the KEGG lysosome pathway (Figure 4C) revealed strong up-regulation of genes encoding subunits of the vacuolar ATPase (V-ATPase), as well as cholesterol trafficking (NPC1), iron transport (MCOLN1) and glycolipid metabolism (GBA). Three of the top seven upregluated genes were ATP6VOA1, ATP6VOA2 and ATP6V1H, all which encode subunits of V-ATPase, an enzyme involved in acidification of intracellular compartments and regulation of inflammatory cytokine production. Notably, inhibitors of VATPase activity increase NF-KB activation and TNF $\alpha$ and IL-12 secretion in macrophages ${ }^{31}$, 32, which would be consistent with increased V-ATPase activity suppressing pro-inflammatory cytokine secretion as observed in our studies.

The top ten down-regulated pathways are shown in Figure 5A. Significantly regulated pathways (nominal $P$ value $<0.01$, Q-value $<0.1$ ) were identified in the KEGG, PID and REACTOME databases as being primarily involved in cell cycle, immune system, gene expression (transcription) and signal transduction (Figure 5B). Closer expression of the repressed gene pathways showed classical inflammatory pathways such as TLR, IL23 and JAK-STAT signalling were down-regulated, which appears to be consistent with reports of inhibitory effects of PC on certain molecules within the JAK-STAT and MAPK/ERK pathways, e.g. p38, and decreased production of inflammatory mediators such as PGE2, IL-1 $\beta$ and nitric oxide. ${ }^{7,9,10,33}$ Within the KEGG JAK-STAT pathway, expression of the pro-inflammatory family of interleukins (e.g. IL12, IL23, IL6, IL28, IL29) as well as JAK2 and STAT4 were all suppressed (Figure 5C), and within the PID IL23 pathway genes encoding similar interleukins as well as CXCL1, CXCL9 and IFNG were all down-regulated (Figure 5D). Other down-regulated pathways were involved in arachidonic acid metabolism (involved in production of eicosanoids), and pathways involved in telomere maintenance and RNA polymerase activity.

Overall, these data confirm previous reports that inflammatory and TLR-linked pathways are repressed in innate immune cells exposed to PC. Our results also suggest that in addition to specific targeting of TLR-linked pathways, a more generalised effect on cellular maintenance and transcriptional activity is evident after exposure of cells to PC. We also provide some evidence of a novel mechanism whereby PC modulates 
inflammatory activity in DCs by modulating the expression of genes involved in V-ATPase and secondmessenger activity. These pathways are intimately connected: adenylyl cyclase, as a promoter of cAMP, plays a critical role in regulating the translocation and function of V-ATPases in the lysosome. ${ }^{34}$ The role of the VATPases in inflammatory cytokine production has been established in several studies, where specific inhibitors of V-ATPase activity increase TNF $\alpha$ production and NF-KB translocation in macrophages ${ }^{31,35}$, as well as promoting the polarization of macrophages towards an anti-inflammatory $\mathrm{M} 2$ phenotype. ${ }^{36}$ The mechanisms behind these effects have not been fully elucidated, but appear to be independent of $\mathrm{pH}$ changes caused by V-ATPase activity. ${ }^{31}$ How PC interacts with V-ATPases has not yet been established. We speculate that in cells involved in phagocytosis, such as DCs and macrophages, PC are trafficked to the lysosome upon endocytosis, as we have shown previously ${ }^{19}$, and subsequently modulate energy metabolism and signalling pathways that result from processing of antigens such as LPS, inhibiting the full activation of the cell and secretion of inflammatory cytokines. Consistent with this, the up-regulation of multiple pathways involved in nutrient metabolism suggests a profound effect on DC metabolic activity during TLR activation. The functional implications of this are not yet clear. However, the importance of immunometabolism in governing the activity of DCs and macrophages during inflammation is becoming increasingly clear, with altered patterns of glucose metabolism and fatty acid oxidation playing a key role in the transition from a resting to activated state following antigen internalisation. ${ }^{37}$. Metabolic reprogramming of the cell during LPS exposure may contribute to the anti-inflammatory properties of PC, and further experiments to explore this are on-going in our laboratory. It will also be of interest to determine whether these effects on nutrient metabolism are specific to phagocytes such as DCs, and are connected to the effects on lysosome function, or may be a more generalised response of cells to PC exposure.

\section{Conclusions}

We have shown for the first time the genome-wide transcriptional changes occurring in human DCs exposed to PC during inflammatory stimulus. Our work provides a foundation for understanding how polyphenols modulate inflammatory activity in human cells. Further work will now focus on understanding how these changes modify the biochemical activity of human DCs and other innate immune cells under inflammatory conditions.

\section{Acknowledgements}

We are grateful to Sara Almeida and Chris Humphrey for assistance, and Lars Andresen and Søren Skov for useful discussions and help with buffy coat collection. This work was supported by the Danish Council for 
Independent Research (DFF-FTP grant \# 12-126630) and the Lundbeck Foundation (Grant \# 14-3670A). AR was supported by the EU Marie Curie Initial Training Network 'LegumePlus' (PITN-GA-2011-289377).

\section{Conflicts of Interest}

There are no conflicts of interest to declare. 


\section{Figure Legends}

\section{Figure 1.}

Chemical structure of Epicatechin and catechin, the monomeric cis-flavan-3-ol isomers giving rise to procyanidin oligomers found in cocoa.

\section{Figure 2.}

Differentially expressed genes identified by microarray in human dendritic cells (DCs) exposed to cocoa procyanidins and lipopolysaccharide (LPS), relative to DCs cultured with only LPS (each $n=3$ ). Shown are the top 20 significantly up-regulated and top 15 down-regulated genes (see Supplementary Material for full list of all regulated genes).

\section{Figure 3.}

A) qPCR validation of selected differentially expressed genes in dendritic cells (DCs) exposed to procyanidins (PC) and lipopolysaccharide (LPS) relative to LPS alone (each $n=3)$. B) Secretion of IL-23 ( $n=3$ ) and CXCL1 $(n=5)$ from DCs exposed to LPS alone and LPS and PC. Shown is the mean \pm S.E.M. \# P<0.1; ${ }^{*} P<0.05 ;{ }^{* *} P<0.01 ;{ }^{* * *} P<0.001$ compared to LPS only by one-way ANOVA or two-tailed t-test.

\section{Figure 4.}

Gene pathways up-regulated by procyanidin (PC) treatment in lipopolysaccharide-exposed dendritic cells (DCs), as determined by gene-set enrichment analysis (GSEA). A) Top ten up-regulated pathways (see Supplementary Material for full list of all differentially regulated pathways), B) Gene ontogeny (GO) terms describing the most enriched gene pathways in PCA-treated DCs, from the KEGG and REACTOME databases C) Significantly up-regulated genes involved in the KEGG Lysosome pathway, as shown by GSEA.

\section{Figure 5.}

Gene pathways down-regulated by procyanidin (PC) treatment in lipopolysaccharide-exposed dendritic cells (DCs), as determined by gene-set enrichment analysis (GSEA). A) Top ten down-regulated pathways (see Supplementary Material for full list of all differentially regulated pathways), B) Gene ontogeny (GO) terms describing the most supressed gene pathways in PC-treated DCs, from the KEGG, PID and REACTOME databases C) Significantly down-regulated genes involved in the KEGG JAK-STAT pathway, as shown by GSEA, D) Significantly down-regulated genes involved in the PID IL-23 pathway, as shown by GSEA. 
Table 1

Primers used for qPCR

\begin{tabular}{|c|c|c|}
\hline Gene & Forward Primer (5' - 3') & Reverse Primer (5' - 3') \\
\hline$A D C Y 3$ & GAGTCACCCCCGATGTCAAC & TTGCCCCAGATGTCGTAGTG \\
\hline CXCL1 & GAACATCCAAAGTGTGAACGTGAAG & TTCAGGAACAGCCACCAGTGAG \\
\hline GUCY1A3 & CAACCGTGCCCATCTGTCAAG & CATTCAGCCGTTCAAACTCTGG \\
\hline$N M B$ & CCGCGGCCCGGGCACAGCC & CGGTGGCCCAGAGGTTGCC \\
\hline PTGS2 & CTTCACGCATCAGTTTTTCAAG & TCACCGTAAATATGATTTAAGTCCAC \\
\hline RPLPO & CCTCGTGGAAGTGACATCGT & CATTCCCCCGGATATGAGGC \\
\hline SPINK1 & TGCTGCCATCTGCCATATGACCC & ATGGCTGAAGTTCTGCGTCCAG \\
\hline
\end{tabular}




\section{References}

1. N. Martinez-Micaelo, N. González-Abuín, A. Ardèvol, M. Pinent and M. T. Blay, Procyanidins and inflammation: Molecular targets and health implications, Biofactors, 2012, 38, 257-265.

2. M.-J. Bak, V. L. Truong, H.-S. Kang, M. Jun and W.-S. Jeong, Anti-Inflammatory Effect of Procyanidins from Wild Grape (Vitis amurensis) Seeds in LPS-Induced RAW 264.7 Cells, Oxidative Medicine and Cellular Longevity, 2013, 2013, 11.

3. H. S. Oz, T. Chen and W. J. de Villiers, Green tea polyphenols and sulfasalazine have parallel antiinflammatory properties in colitis models, Frontiers in Immunology, 2013, 4.

4. M. Miyake, K. Sasaki, K. Ide, Y. Matsukura, K. Shijima and D. Fujiwara, Highly Oligomeric Procyanidins Ameliorate Experimental Autoimmune Encephalomyelitis via Suppression of Th1 Immunity, The Journal of Immunology, 2006, 176, 5797-5804.

5. M.-C. Denis, Y. Desjardins, A. Furtos, V. Marcil, S. Dudonné, A. Montoudis, C. Garofalo, E. Delvin, A. Marette and E. Levy, Prevention of oxidative stress, inflammation and mitochondrial dysfunction in the intestine by different cranberry phenolic fractions, Clinical Science, 2015, 128, 197.

6. Z. T. Bitzer, S. L. Glisan, M. R. Dorenkott, K. M. Goodrich, L. Ye, S. F. O'Keefe, J. D. Lambert and A. P. Neilson, Cocoa procyanidins with different degrees of polymerization possess distinct activities in models of colonic inflammation, The Journal of Nutritional Biochemistry, 2015, 26, 827-831.

7. N. Martinez-Micaelo, N. González-Abuín, M. Pinent, A. Ardévol and M. Blay, Procyanidin B2 inhibits inflammasome-mediated IL-1 $\beta$ production in lipopolysaccharide-stimulated macrophages, Molecular Nutrition \& Food Research, 2015, 59, 262-269.

8. M. Da Silva, G. K. Jaggers, S. V. Verstraeten, A. G. Erlejman, C. G. Fraga and P. I. Oteiza, Large procyanidins prevent bile-acid-induced oxidant production and membrane-initiated ERK1/2, p38, and Akt activation in Caco-2 cells, Free Radical Biology and Medicine, 2012, 52, 151-159.

9. N.-Y. Sung, M.-S. Yang, D.-S. Song, J.-K. Kim, J.-H. Park, B.-S. Song, S.-H. Park, J.-W. Lee, H.-J. Park, J.H. Kim, E.-B. Byun and E.-H. Byun, Procyanidin dimer B2-mediated IRAK-M induction negatively regulates TLR4 signaling in macrophages, Biochemical and Biophysical Research Communications, 2013, 438, 122-128.

10. C. Gentile, M. Allegra, F. Angileri, A. M. Pintaudi, M. A. Livrea and L. Tesoriere, Polymeric proanthocyanidins from Sicilian pistachio (Pistacia vera L.) nut extract inhibit lipopolysaccharideinduced inflammatory response in RAW 264.7 cells, Eur J Nutr, 2012, 51, 353-363.

11. I. Mueller-Harvey, Unravelling the conundrum of tannins in animal nutrition and health, Journal of the Science of Food and Agriculture, 2006, 86, 2010-2037.

12. J. D. Reed, Nutritional toxicology of tannins and related polyphenols in forage legumes, Journal of Animal Science, 1995, 73, 1516-1528.

13. Y. Y. Choy, P. Quifer-Rada, D. M. Holstege, S. A. Frese, C. C. Calvert, D. A. Mills, R. M. LamuelaRaventos and A. L. Waterhouse, Phenolic metabolites and substantial microbiome changes in pig feces by ingesting grape seed proanthocyanidins, Food \& Function, 2014, 5, 2298-2308.

14. S. Jang, J. Sun, P. Chen, S. Lakshman, A. Molokin, J. M. Harnly, B. T. Vinyard, J. J. F. Urban, C. D. Davis and G. Solano-Aguilar, Flavanol-Enriched Cocoa Powder Alters the Intestinal Microbiota, Tissue and Fluid Metabolite Profiles, and Intestinal Gene Expression in Pigs, The Journal of Nutrition, 2016, 146, 673-680.

15. J. Farache, I. Koren, I. Milo, I. Gurevich, K.-W. Kim, E. Zigmond, Glaucia C. Furtado, Sergio A. Lira and G. Shakhar, Luminal Bacteria Recruit CD103+ Dendritic Cells into the Intestinal Epithelium to Sample Bacterial Antigens for Presentation, Immunity, 2013, 38, 581-595.

16. O. Schulz and O. Pabst, Antigen sampling in the small intestine, Trends in Immunology, 2013, 34, 155161.

17. C. L. Scott, C. C. Bain, P. B. Wright, D. Sichien, K. Kotarsky, E. K. Persson, K. Luda, M. Guilliams, B. N. Lambrecht, W. W. Agace, S. W. Milling and A. M. Mowat, CCR2+CD103-intestinal dendritic cells 
develop from DC-committed precursors and induce interleukin-17 production by T cells, Mucosal Immunol, 2015, 8, 327-339.

18. S.-Y. Chang, H.-J. Ko and M.-N. Kweon, Mucosal dendritic cells shape mucosal immunity, Exp Mol Med, 2014, 46, e84.

19. A. R. Williams, E. J. Klaver, L. C. Laan, A. Ramsay, C. Fryganas, R. Difborg, H. Kringel, J. D. Reed, I. Mueller-Harvey, S. Skov, I. van Die and S. M. Thamsborg, Co-operative suppression of inflammatory responses in human dendritic cells by plant proanthocyanidins and products from the parasitic nematode Trichuris suis, Immunology, 2017, 150, 312-328.

20. A. R. Williams, C. Fryganas, A. Ramsay, I. Mueller-Harvey and S. M. Thamsborg, Direct anthelmintic effects of condensed tannins from diverse plant sources against Ascaris suum, PLOS ONE, 2014, 9, e97053.

21. A. Gea, E. Stringano, R. H. Brown and I. Mueller-Harvey, In Situ Analysis and Structural Elucidation of Sainfoin (Onobrychis viciifolia) Tannins for High-Throughput Germplasm Screening, Journal of Agricultural and Food Chemistry, 2011, 59, 495-503.

22. L. Y. Rios, R. N. Bennett, S. A. Lazarus, C. Rémésy, A. Scalbert and G. Williamson, Cocoa procyanidins are stable during gastric transit in humans, The American Journal of Clinical Nutrition, 2002, 76, 11061110.

23. C. Manach, A. Scalbert, C. Morand, C. Rémésy and L. Jiménez, Polyphenols: food sources and bioavailability, The American Journal of Clinical Nutrition, 2004, 79, 727-747.

24. V. G. Tusher, R. Tibshirani and G. Chu, Significance analysis of microarrays applied to the ionizing radiation response, Proceedings of the National Academy of Sciences of the United States of America, 2001, 98, 5116-5121.

25. H. L. E. Midttun, N. Acevedo, P. Skallerup, S. Almeida, K. Skovgaard, L. Andresen, S. Skov, L. Caraballo, I. van Die, C. B. Jørgensen, M. Fredholm, S. M. Thamsborg, P. Nejsum and A. R. Williams, Ascaris Suum Infection Downregulates Inflammatory Pathways in the Pig Intestine In Vivo and in Human Dendritic Cells In Vitro, The Journal of Infectious Diseases, 2018, 217, 310-319.

26. J. D. Nathan, J. Romac, R. Y. Peng, M. Peyton, D. C. Rockey and R. A. Liddle, Protection Against Chronic Pancreatitis and Pancreatic Fibrosis in Mice Over-Expressing Pancreatic Secretory Trypsin Inhibitor, Pancreas, 2010, 39, e24-e30.

27. T. Kambayashi, R. P. A. Wallin and H.-G. Ljunggren, CAMP-elevating agents suppress dendritic cell function, Journal of Leukocyte Biology, 2001, 70, 903-910.

28. D. Giordano, D. M. Magaletti and E. A. Clark, Nitric oxide and cGMP protein kinase (cGK) regulate dendritic-cell migration toward the lymph-node-directing chemokine CCL19, Blood, 2006, 107, 1537.

29. Y. Yu, A. Wu, Z. Zhang, G. Yan, F. Zhang, L. Zhang, X. Shen, R. Hu, Y. Zhang, K. Zhang and F. Wang, Characterization of the GufA subfamily member SLC39A11/Zip11 as a zinc transporter, The Journal of Nutritional Biochemistry, 2013, 24, 1697-1708.

30. I. Stamenkovic, Extracellular matrix remodelling: the role of matrix metalloproteinases, The Journal of Pathology, 2003, 200, 448-464.

31. L. Thomas, Z. Rao, J. Gerstmeier, M. Raasch, C. Weinigel, S. Rummler, D. Menche, R. Müller, C. Pergola, A. Mosig and O. Werz, Selective upregulation of TNF $\alpha$ expression in classically-activated human monocyte-derived macrophages (M1) through pharmacological interference with V-ATPase, Biochemical Pharmacology, 2017, 130, 71-82.

32. I. M. Conboy, D. Manoli, V. Mhaiskar and P. P. Jones, Calcineurin and vacuolar-type H(+)-ATPase modulate macrophage effector functions, Proceedings of the National Academy of Sciences of the United States of America, 1999, 96, 6324-6329.

33. S. Madrigal-Carballo, G. Rodríguez, M. Sibaja, J. D. Reed, A. O. Vila and F. Molina, Chitosomes loaded with cranberry proanthocyanidins attenuate the bacterial lipopolysaccharide-induced expression of iNOS and COX-2 in raw 264.7 macrophages, Journal of Liposome Research, 2009, 19, 189-196.

34. N. Rahman, L. Ramos-Espiritu, T. A. Milner, J. Buck and L. R. Levin, Soluble adenylyl cyclase is essential for proper lysosomal acidification, The Journal of General Physiology, 2016, 148, 325. 
35. A. Bidani and T. A. Heming, Effects of bafilomycin A1 on functional capabilities of LPS-activated alveolar macrophages, Journal of Leukocyte Biology, 1995, 57, 275-281.

36. T. Kimura, S. Nada, N. Takegahara, T. Okuno, S. Nojima, S. Kang, D. Ito, K. Morimoto, T. Hosokawa, Y. Hayama, Y. Mitsui, N. Sakurai, H. Sarashina-Kida, M. Nishide, Y. Maeda, H. Takamatsu, D. Okuzaki, M. Yamada, M. Okada and A. Kumanogoh, Polarization of M2 macrophages requires Lamtor1 that integrates cytokine and amino-acid signals, Nature Communications, 2016, 7, 13130.

37. L. A. J. O'Neill and E. J. Pearce, Immunometabolism governs dendritic cell and macrophage function, The Journal of Experimental Medicine, 2016, 213, 15. 
Figures 